\title{
Almost periodic solution of an impulsive multispecies logarithmic population model
}

Li Wang* and Hui Zhang

\section{"Correspondence:}

mazhilaoma@163.com

Department of Applied

Mathematics, Northwestern

Polytechnical University, Xi'an,

Shaanxi 710072, P.R. China

\begin{abstract}
In this paper, some easily verifiable conditions are derived for the existence of almost periodic solution of an impulsive multispecies logarithmic population model in terms of the continuous theorem of coincidence degree theory, which is rarely applied to studying the existence of almost periodic solution of an impulsive differential equation. Our results generalize previous results by Alzabut, Stamov and Sermutlu. Besides, our technique used in this paper can be applied to study the existence of almost periodic solution of an impulsive differential equation with linear impulsive perturbations.
\end{abstract}

Keywords: coincidence degree theory; almost periodicity; impulsive multispecies logarithmic population model

\section{Introduction}

Many evolutionary processes in nature are characterized by the fact that their states are subject to sudden changes at certain moments and therefore can be described by an impulsive system. Basic theory of impulsive differential equations can be found in the monographs [1-3]. Gopalsamy [4] proposed the following single species logarithmic population model:

$$
N^{\prime}(t)=N(t)(a(t)-b(t) \ln N(t)-c(t) \ln N(t-\tau(t))) .
$$

By utilizing the continuous theorem of coincidence degree theory, the existence of periodic solution and almost periodic solution of this model is investigated in papers [5] and [6], respectively. In paper [7], Alzabut and Abdeljawad consider the existence of periodic solution of the impulsive differential equation

$$
\left\{\begin{array}{l}
N^{\prime}(t)=N^{\prime}(t)(a(t)-b(t) \ln N(t)-c(t) \ln N(t-\tau(t))), \quad t \neq t_{k}, \\
\Delta N\left(t_{k}^{+}\right)=N\left(t_{k}\right)^{1+\gamma_{k}} e^{\delta_{k}}, \quad k=1,2, \ldots
\end{array}\right.
$$

By employing the contraction mapping principle and Gronwall-Bellman inequality, the existence and exponential stability of positive almost periodic solution of this model are obtained in paper [8]. Using the same method as [8], Yang and Li [9] deal with the existence of almost periodic solution of an impulsive two-species logarithmic population model with time-varying delay. By means of the Cauchy matrix, Wang [10] gives sufficient 
conditions for the existence and exponential stability of periodic and almost periodic solution for the delay impulsive logarithmic population:

$$
\left\{\begin{array}{l}
N^{\prime}(t)=N^{\prime}(t)(a(t)-b(t) \ln N(t)-c(t) \ln N(t-\tau(t))), \quad t \neq t_{k}, \\
e^{N\left(t_{k}^{+}\right)}=e^{\left(1+p_{k}\right) N\left(t_{k}\right)+c_{k}}, \quad k=1,2, \ldots
\end{array}\right.
$$

Some scholars are interested in impulsive differential equations or the existence of (almost) periodic solution. Related results can be found in the literature [11-14]. By using fixed point theory and constructing a suitable Lyapunov function, Chen [15] studies the existence, uniqueness and global attractivity of positive periodic solution and almost periodic solution of the delay multispecies logarithmic population model

$$
\begin{aligned}
N_{i}^{\prime}(t)= & N_{i}(t)\left[r_{i}(t)-\sum_{j=1}^{n} a_{i j}(t) \ln N_{j}(t)-\sum_{j=1}^{n} b_{i j}(t) \ln N_{j}\left(t-\tau_{i j}(t)\right)\right. \\
& \left.-\sum_{j=1}^{n} c_{i j}(t) \int_{-\infty}^{t} k_{i j}(t-s) \ln N_{j}(s) d s\right], \quad i=1,2, \ldots, n .
\end{aligned}
$$

In this paper, we consider the impulsive multispecies logarithmic population model

$$
\begin{aligned}
N_{i}^{\prime}(t)= & N_{i}(t)\left[r_{i}(t)-\sum_{j=1}^{n} a_{i j}(t) \ln N_{j}(t)-\sum_{j=1}^{n} b_{i j}(t) \ln N_{j}\left(t-\tau_{i j}(t)\right)\right. \\
& \left.-\sum_{j=1}^{n} c_{i j}(t) \int_{-\infty}^{t} k_{i j}(t-s) \ln N_{j}(s) d s\right], \quad t \neq t_{k}, \\
N_{i}\left(t_{k}^{+}\right)= & \left(1+d_{i k}\right) N_{i}\left(t_{k}\right), \quad i=1,2, \ldots, n, k=1,2, \ldots,
\end{aligned}
$$

where $r_{i}(\cdot), a_{i j}(\cdot), b_{i j}(\cdot), c_{i j}(\cdot), \tau_{i j}(\cdot)$ are nonnegative almost periodic functions in sense of Bohr, $k_{i j}(t-s) \geq 0$ and $\int_{-\infty}^{t} k_{i j}(t-s) d s<+\infty,\left\{d_{i k}\right\}$ is an almost periodic sequence, $\left\{t_{k}\right\}$ is an equipotentially almost periodic sequence, $r=\sup _{t \in R, i, j=1, \ldots, n}\left|\tau_{i, j}(t)\right|, i, j=1, \ldots, n$.

By utilizing the continuous theorem of coincidence degree theory, we obtain some sufficient conditions for the existence of almost periodic solution of Eq. (1.1). Our results generalize previous results obtained in [6] and are easier to verify than the conditions obtained in paper [15].

The continuous theorem of coincidence degree theory has been extensively used to study the existence of periodic solution of a differential equation, regardless of the equation being with impulse or without. Using this theorem, papers [6] and [16] investigate the existence of almost periodic solution of a population model without impulse. To our best knowledge, the continuous theorem has not been used to prove the existence of almost periodic solution of an impulsive multispecies logarithmic population model. Besides, our technique used in this paper can be applied to study the existence of almost periodic solution of an impulsive differential equation with linear impulsive perturbations.

The remaining part of this paper is organized as follows. We present some preliminaries in the next section. In Section 3, by employing the continuous theorem of coincidence degree theory, we establish a criterion for the existence of almost periodic solution of system (1.1). 


\section{Preliminaries}

In this section, some lemmas and definitions, which are of importance in proving our main result in Section 3, will be presented.

Definition 2.1 ([17]) $\varphi(\cdot) \in C\left(R, R^{n}\right)$ is said to be almost periodic in sense of Bohr if $\forall \varepsilon>0$, there exists a relatively dense set $T(\varphi, \varepsilon)$ such that if $\tau \in T(\varphi, \varepsilon)$, then $|\varphi(t)-\varphi(t+\tau)|<\varepsilon$ for all $t \in R$. Denote by ap $\left(R, R^{n}\right)$ all such functions.

Definition $2.2([3])$ Let $\varphi(\cdot)=\left(\varphi_{1}(\cdot), \varphi_{2}(\cdot), \ldots, \varphi_{n}(\cdot)\right)$ be a piecewise continuous function with first kind discontinuities at the points of a fixed sequence $\left\{t_{k}\right\}$. We call $\varphi$ almost periodic if:

(1) $\left\{t_{k}\right\}$ is equipotentially almost periodic, that is, $\forall \varepsilon>0$, there exists a relatively dense set of $\varepsilon$-almost periodic common for any sequences $\left\{t_{k}^{j}\right\}, t_{k}^{j}=t_{k+j}-t_{k}$;

(2) $\forall \varepsilon>0, \exists \delta>0$ such that if the points $t^{\prime}$, $t^{\prime \prime}$ belong to the same interval of continuity and $\left|t^{\prime}-t^{\prime \prime}\right|<\delta$, then $\left|\varphi\left(t^{\prime}\right)-\varphi\left(t^{\prime \prime}\right)\right|<\varepsilon$;

(3) $\forall \varepsilon>0$, there exists a relatively dense set $T(\varphi, \varepsilon)$ such that if $\tau \in T(\varphi, \varepsilon)$, then $|\varphi(t)-\varphi(t+\tau)|<\varepsilon$ for all $t \in R$ which satisfy the condition $\left|t-t_{i}\right|>\varepsilon$, $i=0, \pm 1, \pm 2, \ldots$.

Denote by $\mathcal{A P}\left(R, R^{n}\right)$ all such functions.

Now, we introduce some basic notations. Suppose $f \in \operatorname{ap}\left(R, R^{n}\right)$ or $f \in \mathcal{A P}\left(R, R^{n}\right)$, we use $\Lambda_{f}$ to denote the set of Fourier exponents of $f, \bmod (f)$ to denote the module of $f, m(f)$ to denote the limit mean of $f$. We suppose that $d_{i k}$ and $\tau_{i j}(\cdot)$ in system (1.1) satisfy the following conditions:

(A1) $\prod_{0<t_{k}<t}\left(1+d_{j k}\right), \prod_{0<t_{k}<t-\tau_{i j}(t)}\left(1+d_{j k}\right)$ are positive almost periodic functions, $\inf _{t \in R} \prod_{0<t_{k}<t}\left(1+d_{j k}\right)>0, j=1, \ldots, n$;

(A2) $\int_{-\infty}^{t} k_{i j}(t-s) \ln \prod_{0<t_{k}<s}\left(1+d_{j k}\right) d s$ are almost periodic functions, $i, j=1, \ldots, n$.

Thereinto, the definition of $\prod_{0<t_{k}<t}\left(1+d_{j k}\right)(j=1,2, \ldots, n)$ is as follows:

$$
\prod_{0<t_{k}<t}\left(1+d_{j k}\right)= \begin{cases}1, & t \in\left(-\infty, t_{1}\right], \\ \left(1+d_{j 1}\right) \cdots\left(1+d_{j k}\right), & t \in\left(t_{k}, t_{k+1}\right], k=1,2,3, \ldots\end{cases}
$$

Remark There exist a great deal of functions satisfying assumptions (A1) and (A2). For instance, let $\left\{t_{k}\right\}$ be an arbitrary equipotentially almost periodic sequence, $\tau_{i j}(t)=\tau>0$, $d_{j, k}=d_{j, k+T},\left(1+d_{j, k}\right)>0, k=1,2, \ldots$ and $\left(1+d_{j 1}\right)\left(1+d_{j 2}\right) \ldots\left(1+d_{j T}\right)=1$, then $\prod_{0<t_{k}<t}\left(1+d_{j k}\right)$ is a positive almost periodic function with discontinuous points $t_{k}$, and $\inf _{t \in R} \prod_{0<t_{k}<t}(1+$ $\left.d_{j k}\right)>0 . \prod_{0<t_{k}<t-\tau_{i j}(t)}\left(1+d_{j k}\right)$ is also a positive almost periodic function with discontinuous points $t_{k}+\tau, i, j=1, \ldots, n$.

Besides, we suppose that $k_{i j}(\cdot)$ satisfy $\int_{-\infty}^{t} k_{i j}(t-s) d s=\int_{0}^{+\infty} k_{i j}(u) d u<+\infty$ and

$$
k_{i j}(t)= \begin{cases}0, & t \in\left[0, \sup _{i \in Z} t_{i}^{1}+1\right] \\ \geq 0, & \text { otherwise }\end{cases}
$$

then $\int_{-\infty}^{t} k_{i j}(t-s) \ln \prod_{0<t_{k}<s}\left(1+d_{j k}\right) d s \equiv 0, i, j=1,2, \ldots, n$. Thus, assumption (A2) holds. 
Before studying the existence of strictly positive $\mathcal{A P}$ solutions of system (1.1), we firstly consider the following equation:

$$
\begin{aligned}
y_{i}^{\prime}(t)= & y_{i}(t)\left[r_{i}(t)-\sum_{j=1}^{n} a_{i j}(t) \ln \prod_{0<t_{k}<t}\left(1+d_{j k}\right)-\sum_{j=1}^{n} b_{i j}(t) \ln \prod_{0<t_{k}<t-\tau_{i j}(t)}\left(1+d_{j k}\right)\right. \\
& -\sum_{j=1}^{n} c_{i j}(t) \int_{-\infty}^{t} k_{i j}(t-s) \ln \prod_{0<t_{k}<s}\left(1+d_{j k}\right) d s-\sum_{j=1}^{n} a_{i j}(t) \ln y_{j}(t) \\
& \left.-\sum_{j=1}^{n} b_{i j}(t) \ln y_{j}\left(t-\tau_{i j}(t)\right)-\sum_{j=1}^{n} c_{i j}(t) \int_{-\infty}^{t} k_{i j}(t-s) \ln y_{j}(s) d s\right],
\end{aligned}
$$

where $i=1,2, \ldots, n$. The solutions of Eqs. (1.1) and (2.1) satisfy the following relation.

Lemma 2.3 Suppose that (A1) is satisfied, the following results hold:

(1) If $N_{i}(t) \in \mathcal{A P}(R, R)$ is a positive solution of Eq. (1.1), then $y_{i}(t)=\prod_{0<t_{k}<t}\left(1+d_{i k}\right)^{-1} N_{i}(t)$ is a positive ap solution of Eq. $(2.1), i=1,2, \ldots, n$.

(2) If $y_{i}(t) \in \mathrm{ap}(R, R)$ is a positive solution of $E q$. (2.1), then $N_{i}(t)=\prod_{0<t_{k}<t}\left(1+d_{i k}\right) y_{i}(t)$ is a positive $\mathcal{A P}$ solution of Eq. (1.1), $i=1,2, \ldots, n$.

Proof Since $\prod_{0<t_{k}<t}\left(1+d_{i k}\right) \in \mathcal{A} \mathcal{P}(R, R)$ and $\inf _{t \in R} \prod_{0<t_{k}<t}\left(1+d_{i k}\right)>0, i=1, \ldots, n$, from [3] we know $\prod_{0<t_{k}<t}\left(1+d_{i k}\right)^{-1} \in \mathcal{A P}(R, R)$. Therefore, if $y_{i}(t) \in \operatorname{ap}(R, R)$, then $N_{i}(t)=$ $\prod_{0<t_{k}<t}\left(1+d_{i k}\right) y_{i}(t) \in \mathcal{A} \mathcal{P}(R, R)$; if $N_{i}(t) \in \mathcal{A} \mathcal{P}(R, R)$, combining $N_{i}\left(t_{k}^{+}\right)=\left(1+d_{i k}\right) N_{i}\left(t_{k}\right)$, then $y_{i}(t)=\prod_{0<t_{k}<t}\left(1+d_{i k}\right)^{-1} N_{i}(t) \in \mathrm{ap}(R, R)$. Similar as [18], the rest of the proof of Lemma 2.3 can be obtained easily, we omit it here.

In order to investigate Eq. (2.1), we take $y_{i}(t)=e^{x_{i}(t)}$, Eq. (2.1) can be translated to

$$
\begin{aligned}
x_{i}^{\prime}(t)= & \bar{r}_{i}(t)-\sum_{j=1}^{n} a_{i j}(t) x_{j}(t)-\sum_{j=1}^{n} b_{i j}(t) x_{j}\left(t-\tau_{i j}(t)\right) \\
& -\sum_{j=1}^{n} c_{i j}(t) \int_{-\infty}^{t} k_{i j}(t-s) x_{j}(s) d s,
\end{aligned}
$$

where

$$
\begin{aligned}
\bar{r}_{i}(t)= & r_{i}(t)-\sum_{j=1}^{n} a_{i j}(t) \ln \prod_{0<t_{k}<t}\left(1+d_{j k}\right)-\sum_{j=1}^{n} b_{i j}(t) \ln \prod_{0<t_{k}<t-\tau_{i j}(t)}\left(1+d_{j k}\right) \\
& -\sum_{j=1}^{n} c_{i j}(t) \int_{-\infty}^{t} k_{i j}(t-s) \ln \prod_{0<t_{k}<s}\left(1+d_{j k}\right) d s, \quad i=1,2, \ldots, n .
\end{aligned}
$$

Obviously, if Eq. (2.2) has ap solution, then Eq. (2.1) has strictly positive ap solution. It follows from Lemma 2.3 that Eq. (1.1) has strictly positive $\mathcal{A P}$ solution. Consequently, we mainly study the existence of ap solution of Eq. (2.2). To do so, we firstly summarize a few concepts.

Let $X$ and $Z$ be real Banach spaces, $L: \operatorname{dom} L \subset X \rightarrow Z$ be a linear mapping, $N: X \rightarrow Z$ be a continuous mapping. $L$ is called a Fredholm mapping of index zero if $\operatorname{dim} \operatorname{Ker} L=$ 
$\operatorname{codim} \operatorname{Im} L<\infty$ and $\operatorname{Im} L$ is close in $Z$. If $L$ is a Fredholm mapping of index zero, there are continuous projects $P: X \rightarrow X, Q: Z \rightarrow Z$ such that $\operatorname{Im} P=\operatorname{Ker} L, \operatorname{Im} L=\operatorname{Ker} Q=\operatorname{Im}(I-Q)$. It follows that $L \mid \operatorname{dom} L \cap \operatorname{Ker} P:(I-P) X \rightarrow \operatorname{Im} L$ is invertible. We denote the inverse of that map by $K_{p}$. If $\Omega$ is an open subset of $X$, the mapping $N$ will be called $L$-compact on $\Omega$ if $Q N(\bar{\Omega})$ is bounded and $K_{p}(I-Q) N: \bar{\Omega} \rightarrow X$ is compact. Since $\operatorname{Im} Q$ is isomorphic to $\operatorname{Ker} L$, there exists an isomorphism $J: \operatorname{Im} Q \rightarrow \operatorname{Ker} L$. The following result is proved in [19].

Lemma 2.4 (Continuous theorem) Let $\Omega \subset X$ be an open bounded set, let $L$ be a Fredholm mapping of index zero and $N$ be L-compact on $\bar{\Omega}$. Assume

(1) For each $\lambda \in(0,1)$, every solution $x$ of $L x=\lambda N x$ is such that $x \notin \partial \Omega$;

(2) For each $x \in \operatorname{Ker} L \cap \partial \Omega, Q N x \neq 0$;

(3) $\operatorname{deg}(J Q N, \operatorname{Ker} L \cap \Omega, 0) \neq 0$.

Then $L x=N x$ has at least one solution in $\operatorname{dom} L \cap \bar{\Omega}$.

\section{Main results}

In this section, by means of the continuous theorem of coincidence degree theory, we investigate the existence of strictly positive $\mathcal{A P}$ solution of Eq. (1.1). To do so, we take

$$
\begin{aligned}
X_{1}= & \left\{x=\left(x_{1}, \ldots, x_{n}\right) \in \operatorname{ap}\left(R, R^{n}\right): \bmod \left(x_{i}\right) \subset \bmod (F), \forall \lambda \in \Lambda_{x_{i}}, \alpha_{1}>|\lambda|>\alpha\right\} \cup\{0\}, \\
Z_{1}= & \left\{z=\left(z_{1}, \ldots, z_{n}\right) \in \mathcal{A} \mathcal{P}\left(R, R^{n}\right),\right. \\
& z_{i}(t) \text { are } \mathcal{A} \mathcal{P} \text { functions with discontinuous points } t_{k}, \\
& \left.\bmod \left(z_{i}\right) \subset \bmod (F), \forall \lambda \in \Lambda_{z_{i}}, \alpha_{1}>|\lambda|>\alpha, \sum_{j=1}^{\infty}\left|a\left(\lambda_{j}, z_{i}\right)\right|<+\infty\right\} \cup\{0\}, \\
Z_{2}= & X_{2}=\left\{h=\left(h_{1}, h_{2}, \ldots, h_{n}\right) \in R^{n}\right\},
\end{aligned}
$$

where $\alpha$ and $\alpha_{1}$ are given positive constants, and $F$ is a given almost periodic function in sense of Bohr. Define $X=X_{1} \oplus X_{2}, Z=Z_{1} \oplus Z_{2}$ with the norm $\|\phi\|=\max _{1 \leq i \leq n} \sup _{t \in R}\left|\phi_{i}(t)\right|$, $\phi=\left(\phi_{1}, \phi_{2}, \ldots, \phi_{n}\right) \in X$ or $Z$.

Lemma 3.1 $X$ and $Z$ are Banach spaces equipped with the norm $\|\cdot\|$.

Proof Firstly, we can easily obtain that $X$ is a Banach space, hence we only need to prove that $Z$ is a Banach space. If $\left\{z_{k}=\left(z_{1 k}, \ldots, z_{n k}\right)\right\}_{k} \subset Z_{1}$ converges to $z=\left(z_{1}, \ldots, z_{n}\right)$ uniformly, it follows from the properties of $\mathcal{A P}$ functions that $z \in \mathcal{A P}\left(R, R^{n}\right)$. Similar as [16], the fact $\bmod \left(z_{i}\right) \subset \bmod (F), \forall \lambda \in \Lambda_{z_{i}}, \alpha_{1}>|\lambda|>\alpha$ can be obtained easily. Now, we assert that $\sum_{j=1}^{\infty}\left|a\left(\lambda_{j}, z_{i}\right)\right|<+\infty, i=1, \ldots, n$. If not, $\forall M>0, \exists m>0$ and $\bar{i}$ such that

$$
\sum_{j=1}^{m}\left|a\left(\lambda_{j}, z_{\bar{i}}\right)\right|>M .
$$

Since $\left\{z_{k}\right\}$ is a Cauchy sequence, for $1 / 2^{m}, \exists K$ such that for any $\bar{m}, \bar{n}>K$,

$$
\left|a\left(\lambda, z_{\bar{i}, \bar{m}}\right)-a\left(\lambda, z_{\bar{i}, \bar{n}}\right)\right|=\left|\lim _{T \rightarrow+\infty} \frac{1}{2 T} \int_{-T}^{T}\left(z_{\bar{i}, \bar{m}}(t)-z_{\bar{i}, \bar{n}}(t)\right) e^{-i \lambda t} d t\right|<\frac{1}{2^{m}}, \quad \forall \lambda \in R .
$$


Since $z_{k}$ converges to $z$ uniformly,

$$
\text { for } \lambda_{l} \in \Lambda_{z_{\bar{i}}} \text { and } \frac{1}{2^{l}}, \exists n_{l}>K \text {, s.t., }\left|a\left(\lambda_{l}, z_{\bar{i}}^{-}\right)\right|<\left|a\left(\lambda_{l}, z_{\bar{i}, n_{l}}\right)\right|+\frac{1}{2^{l}}, l=1,2, \ldots, m \text {. }
$$

Since $n_{1}, n_{2}, \ldots, n_{m}>K$, from $(3.2)$

$$
\left|a\left(\lambda_{j}, z_{\bar{i}, n_{j}}\right)\right|<\left|a\left(\lambda_{j}, z_{\bar{i}, n_{1}}\right)\right|+\frac{1}{2^{m}}, \quad j=1,2, \ldots, m
$$

Hence, combining (3.1)-(3.4)

$$
\begin{aligned}
M & <\sum_{j=1}^{m}\left|a\left(\lambda_{j}, z_{i}\right)\right|<\sum_{j=1}^{m}\left|a\left(\lambda_{j}, z_{\bar{i}, n_{j}}\right)\right|+\frac{1}{2^{j}}<\sum_{j=1}^{m}\left|a\left(\lambda_{j}, z_{i, n_{j}}\right)\right|+1 \\
& <\sum_{j=1}^{m}\left|a\left(\lambda_{j}, z_{\bar{i}, n_{1}}\right)\right|+1+\frac{m}{2^{m}}<\sum_{j=1}^{\infty}\left|a\left(\lambda_{j}, z_{i, n_{1}}\right)\right|+3<+\infty .
\end{aligned}
$$

The arbitrariness of $M$ leads to a contradiction, so the assertion holds. Therefore, $Z_{1}$ is a Banach space. Similarly, we can obtain that $Z$ is a Banach space. The proof is complete.

Lemma 3.2 Let

$$
L: X \rightarrow Z, \quad L\left(x_{1}, x_{2}, \ldots, x_{n}\right)=\left(\frac{d x_{1}}{d t}, \frac{d x_{2}}{d t}, \ldots, \frac{d x_{n}}{d t}\right)
$$

then L is a Fredholm mapping of index zero.

Proof Obviously, $\operatorname{Ker} L=X_{2}$. We prove $\operatorname{Im} L=Z_{1}$. Firstly, for any $\varphi_{1}+\varphi_{2}=\varphi \in \operatorname{Im} L \subset Z$, $\varphi_{1} \in Z_{1}, \varphi_{2} \in Z_{2}$, since $\int_{0}^{t} \varphi(s) d s \in \operatorname{ap}\left(R, R^{n}\right)$, that is, $\int_{0}^{t} \varphi_{1}(s) d s+\int_{0}^{t} \varphi_{2}(s) d s \in \operatorname{ap}\left(R, R^{n}\right)$. From [20] we know $\int_{0}^{t} \varphi_{1}(s) d s \in \operatorname{ap}\left(R, R^{n}\right)$, then $\varphi_{2}=0$. Hence $\varphi \in Z_{1}, \operatorname{Im} L \subset Z_{1}$. Secondly, for any $\varphi=\left(\varphi_{1}, \varphi_{2}, \ldots, \varphi_{n}\right) \in Z_{1}$, without loss of generality, we suppose $\varphi \neq 0$. Since $\int_{0}^{t} \varphi(s) d s \in \operatorname{ap}\left(R, R^{n}\right)$, furthermore

$$
\Lambda_{\int_{0}^{t} \varphi_{i}(s) d s-m\left(\int_{0}^{t} \varphi_{i}(s) d s\right)}=\Lambda_{\varphi_{i}}, \quad i=1,2, \ldots, n,
$$

then $\int_{0}^{t} \varphi_{i}(s) d s-m\left(\int_{0}^{t} \varphi_{i}(s) d s\right)$ is the primitive of $\varphi_{i}$ in $X, \varphi \in \operatorname{Im} L$. Therefore, $Z_{1} \subset \operatorname{Im} L$. To sum up, $Z_{1}=\operatorname{Im} L$. Besides, one can easily show that $\operatorname{Im} L$ is closed in $Z$

$$
\operatorname{dim} \operatorname{Ker} L=n=\operatorname{codim} \operatorname{Im} L
$$

Therefore, $L$ is a Fredholm mapping of index zero.

Remark If $f \in \operatorname{ap}\left(R, R^{n}\right)$ and $\forall \lambda \in \Lambda_{f},|\lambda|>\alpha>0$, then $f$ has an ap primitive function. It does not hold for an $\mathcal{A P}$ function. From [20] we know that if $f \in \mathcal{A P}\left(R, R^{n}\right), \forall \lambda \in \Lambda_{f}$, $\alpha_{1}>|\lambda|>\alpha>0, \sum_{i=1}^{\infty}\left|a\left(\lambda_{i}, f\right)\right|<+\infty$, then $f$ has an $\mathcal{A P}$ primitive function. That is the reason why in Lemma 3.1 we take $Z_{1}$ like that. 
Set

$$
\begin{aligned}
& N: X \rightarrow Z, \quad N\left(x_{1}, x_{2}, \ldots, x_{n}\right)=\left(N x_{1}, N x_{2}, \ldots, N x_{n}\right), \\
& Q: Z \rightarrow Z, \quad Q\left(z_{1}, z_{2}, \ldots, z_{n}\right)=\left(m\left(z_{1}\right), m\left(z_{2}\right), \ldots, m\left(z_{n}\right)\right), \\
& P: X \rightarrow X, \quad P\left(x_{1}, x_{2}, \ldots, x_{n}\right)=\left(m\left(x_{1}\right), m\left(x_{2}\right), \ldots, m\left(x_{n}\right)\right),
\end{aligned}
$$

where

$$
N x_{i}(t)=\bar{r}_{i}(t)-\sum_{j=1}^{n} a_{i j}(t) x_{j}(t)-\sum_{j=1}^{n} b_{i j}(t) x_{j}\left(t-\tau_{i j}(t)\right)-\sum_{j=1}^{n} c_{i j}(t) \int_{-\infty}^{t} k_{i j}(t-s) x_{j}(s) d s,
$$

then we have the following.

Lemma 3.3 $N$ is L-compact on $\bar{\Omega}(\Omega$ is an open, bounded subset of $X)$.

Proof Firstly, it is easy to show that $P$ and $Q$ are continuous projectors such that

$$
\operatorname{Im} P=\operatorname{Ker} L, \quad \operatorname{Im} L=\operatorname{Im}(I-Q)=\operatorname{Ker} Q,
$$

where $I$ is an identity mapping. Hence $L \mid \operatorname{dom} L \cap \operatorname{Ker} P:(I-P) X \rightarrow \operatorname{Im} L$ is invertible. We denote the inverse of that map by $K_{p} . K_{p}: \operatorname{Im} L \rightarrow \operatorname{Ker} P \cap \operatorname{Dom} L$ has the form

$$
\begin{aligned}
K_{p} z & =K_{p}\left(z_{1}, z_{2}, \ldots, z_{n}\right) \\
& =\left(\int_{0}^{t} z_{1}(s) d s-m\left(\int_{0}^{t} z_{1}(s) d s\right), \ldots, \int_{0}^{t} z_{n}(s) d s-m\left(\int_{0}^{t} z_{n}(s) d s\right)\right) .
\end{aligned}
$$

Thus,

$$
\begin{aligned}
& Q N x=\left(Q N x_{1}, \ldots, Q N x_{n}\right), \\
& K_{p}(I-Q) N x=\left(f\left(x_{1}(t)\right)-Q f\left(x_{1}(t)\right), \ldots, f\left(x_{n}(t)\right)-Q f\left(x_{n}(t)\right)\right),
\end{aligned}
$$

where

$$
\begin{aligned}
f\left(x_{i}(t)\right)= & \int_{0}^{t}\left(N x_{i}(s)-Q N x_{i}(s)\right) d s, \\
Q N x_{i}(t)= & m\left(\bar{r}_{i}(t)-\sum_{j=1}^{n} a_{i j}(t) x_{j}(t)-\sum_{j=1}^{n} b_{i j}(t) x_{j}\left(t-\tau_{i j}(t)\right)\right. \\
& \left.-\sum_{j=1}^{n} c_{i j}(t) \int_{-\infty}^{t} k_{i j}(t-s) x_{j}(s) d s\right) .
\end{aligned}
$$

Obviously, $Q N$ and $(I-Q) N$ are continuous. In fact, $K_{p}$ is also continuous. For any $z=\left(z_{1}, \ldots, z_{n}\right) \in Z_{1}$ and for any $1>\varepsilon>0$, let $l(\varepsilon)$ denote the inclusion interval of $T(F, \varepsilon)$. Suppose $z=\left(z_{1}, z_{2}, \ldots, z_{n}\right) \in Z_{1}=\operatorname{Im} L$, then $\int_{0}^{t} z(s) d s \in \operatorname{ap}\left(R, R^{n}\right)$. Since

$$
\Lambda_{\int_{0}^{t} z_{i}(s) d s} \backslash\{0\}=\Lambda_{\int_{0}^{t} z_{i}(s) d s-m\left(\int_{0}^{t} z_{i}(s) d s\right)}=\Lambda_{z_{i}}, \quad i=1,2, \ldots, n,
$$


and $\bmod \left(z_{i}\right) \subset \bmod (F)$, then $\bmod \left(\int_{0}^{t} z_{i}(s) d s\right) \subset \bmod (F)$. Therefore, there exists $0<\delta<\varepsilon$ such that $T(F, \delta) \subset T\left(\int_{0}^{t} z_{i}(s) d s, \varepsilon\right)$. Let $l$ be the inclusion interval of $T(F, \delta)$, then $l \geq l(\varepsilon)$. For any $t \notin[0, l]$, there exists $\xi \in T(F, \delta) \subset T\left(\int_{0}^{t} z_{i}(s) d s, \varepsilon\right)$ such that $t+\xi \in[0, l]$. Hence, by the definition of almost periodic function, we have

$$
\begin{aligned}
\sup _{t \in R}\left|\int_{0}^{t} z_{i}(s) d s\right| \leq & \sup _{t \in[0, l]}\left|\int_{0}^{t} z_{i}(s) d s\right|+\sup _{t \notin[0, l]}\left|\int_{0}^{t} z_{i}(s) d s-\int_{0}^{t+\xi} z_{i}(s) d s\right| \\
& +\sup _{t \notin[0, l]}\left|\int_{0}^{t+\xi} z_{i}(s) d s\right| \\
\leq & 2 \sup _{t \in[0, l]} \int_{0}^{t}\left|z_{i}(s)\right| d s+\sup _{t \notin[0, l]}\left|\int_{0}^{t} z_{i}(s) d s-\int_{0}^{t+\xi} z_{i}(s) d s\right| \\
\leq & 2 \int_{0}^{l}\left|z_{i}(s)\right| d s+\varepsilon .
\end{aligned}
$$

Hence, we can conclude that $K_{p}$ is continuous, and consequently, $K_{p}(I-Q) N$ is also continuous. In addition, we also have $K_{p}(I-Q) N$ is uniformly bounded in $\bar{\Omega}, Q N(\bar{\Omega})$ is bounded and $K_{p}(I-Q) N$ is equicontinuous in $\bar{\Omega}$. Since $(I-Q) N x \in Z_{1}=\operatorname{Im} L$ and $\Lambda_{K_{p}(I-Q) N x}=\Lambda_{(I-Q) N x}, \forall x \in \Omega$, then $\bmod \left(K_{p}(I-Q) N x\right)=\bmod ((I-Q) N x) \subset \bmod (F)$. For any $\varepsilon>0, \exists \delta>0$ such that $T(F, \delta) \subset T\left(K_{p}(I-Q) N x, \varepsilon\right), \forall x \in \Omega$, hence $K_{p}(I-Q) N$ is equialmost periodic in $\Omega$, According to [21], we can conclude that $\overline{K_{p}(I-Q) N \bar{\Omega}}$ is compact, thus $N$ is $L$-compact on $\bar{\Omega}$.

Noticing Lemmas 3.1-3.3, for Eq. (1.1), we have the following result.

Theorem 3.4 If (A1) and (A2) are satisfied, $m\left(\sum_{j=1}^{n}\left(a_{i j}(t)+b_{i j}(t)+c_{i j}(t) \int_{-\infty}^{t} k_{i j}(t-s) d s\right)\right) \neq$ $0, i=1,2, \ldots, n$, then Eq. (1.1) has at least one strictly positive almost periodic solution.

Proof From the analysis above, we know that in order to prove the existence of strictly positive $\mathcal{A P}$ solution of Eq. (1.1), we only need to investigate the existence of ap solution of Eq. (2.2).

Define the isomorphism $J: \operatorname{Im} Q \rightarrow \operatorname{Ker} L$ as an identity mapping. We search for an appropriate bounded open subset $\Omega$ for the application of Lemma 2.4. Corresponding to the operator equation $L x=\lambda N x, \lambda \in(0,1)$, we have

$$
\begin{aligned}
x_{i}^{\prime}(t)= & \lambda\left(\bar{r}_{i}(t)-\sum_{j=1}^{n} a_{i j}(t) x_{j}(t)-\sum_{j=1}^{n} b_{i j}(t) x_{j}\left(t-\tau_{i j}(t)\right)\right. \\
& \left.-\sum_{j=1}^{n} c_{i j}(t) \int_{-\infty}^{t} k_{i j}(t-s) x_{j}(s) d s\right), \quad i=1,2, \ldots, n .
\end{aligned}
$$

If $x \in X$ is a solution of system (3.5), taking the limit mean for system (3.5), we obtain

$$
\begin{gathered}
m\left(\bar{r}_{i}(t)\right)=m\left(\sum_{j=1}^{n}\left(a_{i j}(t) x_{j}(t)+b_{i j}(t) x_{j}\left(t-\tau_{i j}(t)\right)+c_{i j}(t) \int_{-\infty}^{t} k_{i j}(t-s) x_{j}(s) d s\right)\right), \\
i=1,2, \ldots, n,
\end{gathered}
$$


then

$$
\begin{aligned}
& \sup _{t \in R} x_{i}(t) \geq \frac{m\left(\bar{r}_{i}(t)\right)}{m\left(\sum_{j=1}^{n}\left(a_{i j}(t)+b_{i j}(t)+c_{i j}(t) \int_{-\infty}^{t} k_{i j}(t-s) d s\right)\right)}, \quad i=1,2, \ldots, n, \\
& \inf _{t \in R} x_{i}(t) \leq \frac{m\left(\bar{r}_{i}(t)\right)}{m\left(\sum_{j=1}^{n}\left(a_{i j}(t)+b_{i j}(t)+c_{i j}(t) \int_{-\infty}^{t} k_{i j}(t-s) d s\right)\right)}, \quad i=1,2, \ldots, n .
\end{aligned}
$$

Thus, there exists at least one $t^{*} \in R$ such that

$$
\left|x_{i}\left(t^{*}\right)\right| \leq \frac{\left|m\left(\bar{r}_{i}(t)\right)\right|}{\left|m\left(\sum_{j=1}^{n}\left(a_{i j}(t)+b_{i j}(t)+c_{i j}(t) \int_{-\infty}^{t} k_{i j}(t-s) d s\right)\right)\right|}+1, \quad i=1,2, \ldots, n .
$$

Since $L x \in Z_{1}$, by a similar argument as in Lemma 3.3 we can derive that

$$
\begin{aligned}
\left\|x_{i}\right\| & =\sup _{t \in R}\left|x_{i}(t)\right| \leq\left|x_{i}\left(t^{*}\right)\right|+\sup _{t \in R}\left|\int_{t^{*}}^{t} x_{i}^{\prime}(s) d s\right| \\
& \leq\left|x_{i}\left(t^{*}\right)\right|+2 \int_{t^{*}}^{t^{*}+l}\left|x_{i}^{\prime}(s)\right| d s+1, \quad i=1,2, \ldots, n .
\end{aligned}
$$

Take $\bar{\tau} \in[l, 2 l] \cap T(F, \delta) \subset T\left(\int_{0}^{t} x_{i}^{\prime}(s) d s, \varepsilon\right)$, then

$$
\left|\int_{0}^{t^{*}} x_{i}^{\prime}(s) d s-\int_{0}^{t^{*}+\bar{\tau}} x_{i}^{\prime}(s) d s\right|=\left|\int_{t^{*}}^{t^{*}+\bar{\tau}} x_{i}^{\prime}(s) d s\right| \leq \varepsilon \leq 1, \quad i=1,2, \ldots, n .
$$

Integrating system (3.5) over the interval $\left[t^{*}, t^{*}+\bar{\tau}\right]$, we obtain

$$
\begin{aligned}
& \lambda \int_{t^{*}}^{t^{*}+\bar{\tau}}\left|\sum_{j=1}^{n}\left(a_{i j}(s) x_{j}(s)+b_{i j}(s) x_{j}\left(s-\tau_{i j}(s)\right)+c_{i j}(s) \int_{-\infty}^{s} k_{i j}(s-u) x_{j}(u) d u\right)\right| d s \\
& \quad \leq \lambda \int_{t^{*}}^{t^{*}+\bar{\tau}}\left|\bar{r}_{i}(s)\right| d s+1 .
\end{aligned}
$$

Consequently,

$$
\begin{aligned}
& \int_{t^{*}}^{t^{*}+l}\left|x_{i}^{\prime}(s)\right| d s \\
& \leq \int_{t^{*}}^{t^{*}+\bar{\tau}}\left|x_{i}^{\prime}(s)\right| d s \leq \lambda \int_{t^{*}}^{t^{*}+\bar{\tau}}\left|\bar{r}_{i}(s)\right| d s \\
& \quad+\lambda \int_{t^{*}}^{t^{*}+\bar{\tau}}\left|\sum_{j=1}^{n}\left(a_{i j}(s) x_{j}(s)+b_{i j}(s) x_{j}\left(s-\tau_{i j}(s)\right)+c_{i j}(s) \int_{-\infty}^{s} k_{i j}(s-u) x_{j}(u) d u\right)\right| d s \\
& \leq 2 \int_{t^{*}}^{t^{*}+\bar{\tau}}\left|\bar{r}_{i}(s)\right| d s+1 .
\end{aligned}
$$

Combining (3.6)-(3.8), we obtain

$$
\left\|x_{i}\right\| \leq M_{1}, \quad i=1,2, \ldots, n,
$$


where

$$
M_{1}=\frac{4\left|m\left(\bar{r}_{i}(t)\right)\right|}{\left|m\left(\sum_{j=1}^{n}\left(a_{i j}(t)+b_{i j}(t)+c_{i j}(t) \int_{-\infty}^{t} k_{i j}(t-s) d s\right)\right)\right|}+4+4 \int_{t^{*}}^{t^{*}+\bar{\tau}}\left|\bar{r}_{i}(s)\right| d s .
$$

Take $\Omega=\left\{x \in X,\|x\| \leq M_{1}+2\right\}$, then it is clear that $\Omega$ verifies all the requirements in Lemma 2.4, hence Eq. (2.2) has at least one almost periodic solution in $\Omega$ and the proof is complete.

Remark In this paper, we investigate the existence of almost periodic solution of Eq. (1.1) in terms of the continuous theorem of coincidence degree theory. The novelty of this paper is not only the method but also the results. In fact, if $d_{i k}=0, c_{i j}=0, i=j=1$, Eq. (1.1) can be rewritten as

$$
N^{\prime}(t)=N(t)[r(t)-a(t) \ln N(t)-b(t) \ln N(t-\tau(t))] .
$$

Paper [6] obtains the existence of almost periodic solution of the above equation based on the assumptions $m(a(t)+b(t)) \neq 0$. Obviously, our results generalize previous results obtained in paper [6].

\section{Competing interests}

The authors declare that they have no competing interests.

\section{Authors' contributions}

All authors contributed equally and significantly in writing this article. All authors read and approved the final manuscript.

\section{Acknowledgements}

The authors would like to thank the Editor and the referees for their constructive suggestions on improving the presentation of the paper. This work was supported by the Fundamental Research Funds for the Central Universities (3102014JC-Q01087), the National Natural Science Foundation of China (No. 31300310).

Received: 9 October 2014 Accepted: 29 January 2015 Published online: 25 March 2015

\section{References}

1. Bainov, DD, Simeonov, PS: Impulsive Differential Equations: Periodic Solution and Applications. Longman, London (1993)

2. Lakshmikantham, V, Bainov, DD, Simeonov, PS: Theory of Impulsive Differential Equations. World Scientific, Singapore (1989)

3. Samoilenko, AM, Perestyuk, NA: Impulsive Differential Equations. World Scientific, Singapore (1995)

4. Gopalsamy, K: Stability and Oscillation in Delay Differential Equations of Population Dynamics. Kluwer Academic, Boston (1992)

5. Li, YK: Attractivity of a positive periodic solution for all other positive solution in a delay population model. Appl. Math. 12, 279-282 (1997) (in Chinese)

6. Alzabut, JO, Stamov, GT, Sermutlu, E: Positive almost periodic solutions for a delay logarithmic population model. Math Comput. Model. 53, 161-167 (2011)

7. Alzabut, JO, Abdeljawad, T: Existence and global attractivity of impulsive delay logarithmic model of population dynamics. Appl. Math. Comput. 198, 463-469 (2008)

8. Alzabut, JO, Stamov, GT, Sermutlu, E: On almost periodic solutions for an impulsive delay logarithmic population model. Math. Comput. Model. 51, 625-631 (2010)

9. Yang, BX, Li, JL: An almost periodic solution for an impulsive two-species logarithmic population model with time-varying delay. Math. Comput. Model. 55, 1963-1968 (2012)

10. Wang, Q, Zhang, HY, Wang, Y: Existence and stability of positive almost periodic solutions and periodic solutions for a logarithmic population model. Nonlinear Anal. 72, 4384-4389 (2010)

11. Chen, DZ, Dai, BX: Periodic solution of second order impulsive delay differential systems via variational method. Appl. Math. Lett. 38, 61-66 (2014)

12. Li, Z, Han, MA, Chen, FD: Almost periodic solutions of a discrete almost periodic logistic equation with delay. Appl. Math. Comput. 232, 743-751 (2014)

13. Li, XD, Song, SJ: Impulsive control for existence, uniqueness and global stability of periodic solutions of recurrent neural networks with discrete and continuously distributed delays. IEEE Trans. Neural Netw. 24, 868-877 (2013) 
14. Li, XD, Bohner, M: An impulsive delay differential inequality and applications. Comput. Math. Appl. 64, 1875-1881 (2012)

15. Chen, FD: Periodic solutions and almost periodic solutions for a delay multispecies logarithmic population model. Appl. Math. Comput. 171, 760-770 (2005)

16. Xie, Y, Li, X: Almost periodic solutions of single population model with hereditary effects. Appl. Math. Comput. 203, 690-697 (2008)

17. Zhang, C: Almost Periodic Type Function and Ergodicity. Science Press, Beijing (2003)

18. He, M, Chen, F, Li, Z: Almost periodic solution of an impulsive differential equation model of plankton allelopathy. Nonlinear Anal. 11, 2296-2301 (2010)

19. Gaines, RE, Mawhin, JL: Coincidence Degree and Nonlinear Differential Equation. Springer, Berlin (1977)

20. Wang, L, Yu, M: Favard's theorem of piecewise continuous almost periodic functions and its application. J. Math. Anal. Appl. 413, 35-46 (2014)

21. Corduneanu, C: Almost Periodic Functions. Chelsea, New York (1989)

Submit your manuscript to a SpringerOpen ${ }^{\circ}$ journal and benefit from:

- Convenient online submission

Rigorous peer review

- Immediate publication on acceptance

- Open access: articles freely available online

- High visibility within the field

- Retaining the copyright to your article

Submit your next manuscript at $\gg$ springeropen.com 